\title{
Impact des programmes de physique au lycée sur la première année d'enseignement supérieur Une enquête de la SFP
}

Suite au changement des programmes de physique au lycée, la commission enseignement de la Société Française de Physique (SFP) a mené en 2014 une enquête auprès des enseignants du supérieur. Malgré un comportement plus actif des étudiants, les collègues ont constaté des difficultés à résoudre des problèmes de physique. Ceci est dû à un manque de rigueur dans le raisonnement et au fait que nombre de concepts ne sont pas maîtrisés. L'absence de liens entre physique et mathématiques dans les programmes ne fait que renforcer ces tendances. Malgré un élargissement de leur culture scientifique, les connaissances des étudiants sont très cloisonnées et non consolidées.

Les résultats complets de l'enquête ont été publiés dans Le Bup, 972 (2015) 319-348.

\section{Contexte de l'enquête}

Les nouveaux programmes du lycée sont entrés en vigueur en septembre 2010 pour la classe de seconde, en 2011 pour la classe de première et en 2012 pour la terminale. Cette réforme a concerné toutes les filières, générales et technologiques. L'enseignement supérieur a ainsi accueilli à la rentrée 2013 des lycéens ayant suivi ces nouveaux programmes. La commission enseignement de la Société Française de Physique (SFP) a alors décidé, à la fin de l'année scolaire 2013-2014, de consulter les enseignants de première année de l'enseignement supérieur (classes préparatoires, IUT, Licence). Une enquête a été mise en ligne du 24 juin au 25 septembre 2014. Elle avait pour objectif de mettre en évidence le profil des étudiants au bout d'un an d'études supérieures, tant sur le plan des connaissances que sur celui des compétences acquises. Grâce à ces résultats, la SFP pourra agir avec d'autres associations $\left(\mathrm{UdPPC}^{(\mathrm{a})}\right.$, UPS $\left.{ }^{(\mathrm{b})}\right)$ pour faire évoluer les programmes, en y intégrant les prérequis nécessaires aux lycéens pour des études supérieures réussies.

\section{Contenu et déroulement de l'enquête}

Nous avons posé 36 questions autour des thèmes suivants :

- le profil des enseignants interrogés (ancienneté, domaines enseignés, filières d'enseignement);

- le profil des étudiants ;

- les connaissances en physique et en mathématiques et leur mobilisation ;

- le raisonnement et la rigueur ;

- les aptitudes calculatoires ;

- la résolution de problèmes ;

- la prise d'initiative et la participation ;

- les aptitudes expérimentales ;

- la démarche et la culture scientifiques ;

- les aptitudes à communiquer (oral, écrit) ;
- la préparation aux études supérieures ;

- la pratique des enseignants du supérieur, suite à ces changements de programme (exploitation des connaissances acquises au lycée, modification de l'enseignement).

Nous présentons dans cet article un résumé des 268 réponses reçues aux 36 questions de l'enquête [1]. Toutes les réponses ont été faites de manière anonyme. La répartition des répondants était la suivante : 35\% d'enseignants de première année de classes préparatoires (CPGE), 15\% d'enseignants d'IUT, 50\% d'enseignants de Licence première année.

\section{Évolution des connaissances et des aptitudes des étudiants}

\section{Connaissances en physique et en mathématiques}

Une large majorité des personnes interrogées, quelle que soit la filière dans laquelle ils enseignent, souligne une modification dans les connaissances en physique et en mathématiques (figs 1 et 2).

En physique, le plus gros changement concerne l'électricité, pour laquelle tout savoir-faire a quasiment disparu, mais aussi le processus de la mesure où le même déclin des connaissances est observé, et à un moindre degré la mécanique. La notion de modélisation (au sens de modèle théorique, à la fois descriptif et prédictif), pourtant considérée comme un des objectifs de la réforme, semble totalement étrangère aux étudiants.

En mathématiques, un élément souligné est la non-maitrise des calculs de base, par exemple en trigonométrie ou dans la manipulation des fractions. En analyse, les calculs de dérivation sont souvent très laborieux, alors que l'intégration est « quasiment insurmontable ». L'absence totale de connaissances sur les équations différentielles pose problème aux étudiants. En géométrie, leurs acquis ont fortement diminué, et la manipulation des vecteurs est 


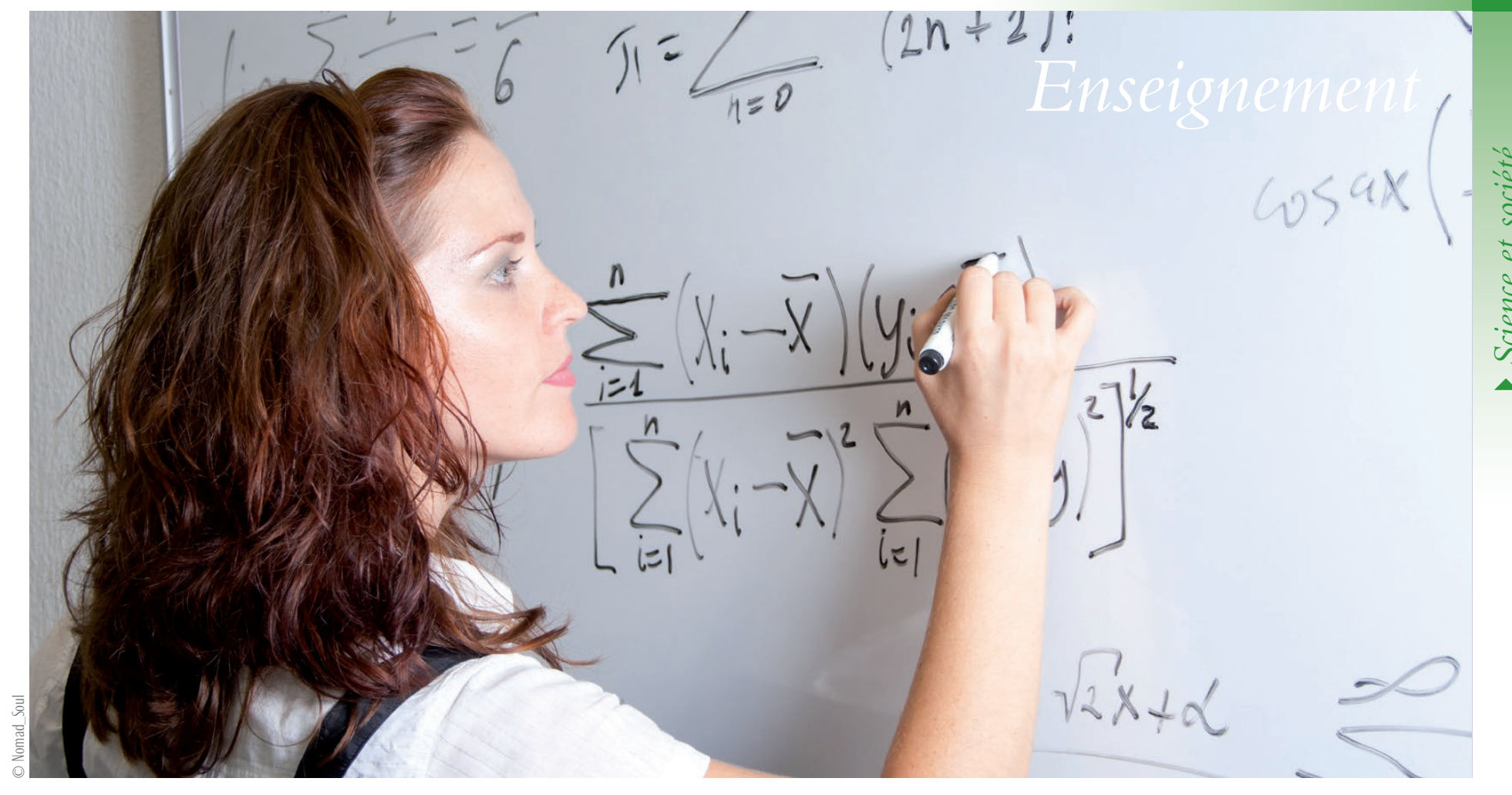

source de grosses difficultés. Les étudiants ont aussi du mal avec les outils statistiques. Leurs lacunes les incitent à utiliser à outrance la calculatrice et l'ordinateur.

Deux réponses, à première vue contradictoires, émergent de l'enquête. D'une part, les répondants, quelle que soit la filière, mettent en avant l'élargissement de la culture scientifique des étudiants. D'autre part, ils soulignent le cloisonnement bien plus grand entre les connaissances acquises, ce qui empêche de les mettre en relation entre elles. Les étudiants n'ont pas une vision globale des différents domaines de la physique.

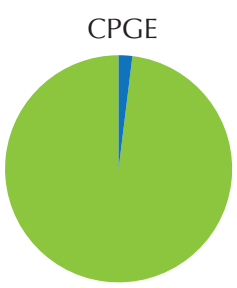

oui : $97,8 \%$ non : $2,2 \%$

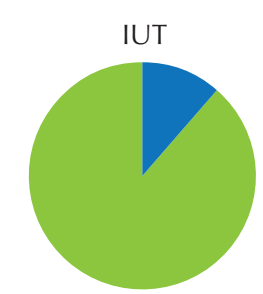

oui : $88,6 \%$ non : $11,4 \%$

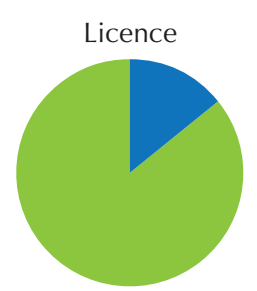

oui : $85,8 \%$ non : $14,2 \%$
1. Répartition des avis des répondants à la question :

"Avez-vous identifié des modifications dans les connaissances des étudiants en physique par rapport aux années précédentes ? »

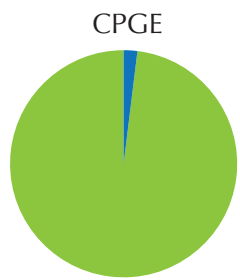

oui : $97,8 \%$ non : $2,2 \%$

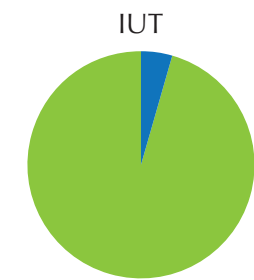

oui : $95,5 \%$ non : $4,5 \%$

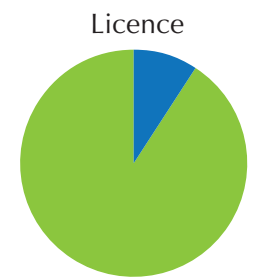

oui : $90,6 \%$ non : $9,4 \%$
2. Répartition des avis des répondants à la question :

" Avez-vous identifié des modifications dans les connaissances des étudiants en mathématiques par rapport aux années précédentes ? ”

\section{Rigueur et raisonnement}

Les avis sont unanimes, la rigueur a fortement diminué. Certains répondants signalent toutefois que cette tendance n'est pas seulement liée à la dernière réforme. Elle l'est aussi au manque d'habitude à modéliser mathématiquement les problèmes étudiés (qui lui, s'est fortement accru avec la réforme). Pour un certain nombre d'étudiants, la physique se réduit à l'étude de documents. Sur l'aptitude au raisonnement, le constat est plus partagé : le raisonnement qualitatif et la compréhension par l'analyse dimensionnelle ont progressé. Les étudiants ont davantage d'intuition et de capacité à proposer des idées, ils prennent plus d'initiatives, mais butent dès qu'il faut approfondir... Ils ont du mal à comprendre qu'une suite de calculs sans explications ne saurait constituer un raisonnement.

\section{Aptitudes calculatoires}

Une grande majorité des enseignants a noté une diminution des aptitudes calculatoires des étudiants. Il semble que la réforme actuelle conduise à l'accentuation d'une tendance installée depuis plusieurs années : la capacité des étudiants à mener un calcul même léger se dégrade, ce qui conduit souvent à un découragement rapide. Ceci renvoie à la question des connaissances en mathématiques. En CPGE, on constate de plus grandes difficultés par rapport aux élèves de l'année précédente.

\section{Aptitudes à la résolution de problèmes}

Un tiers des répondants, toutes filières confondues, constate une baisse des aptitudes à résoudre des problèmes de physique. Certains enseignants de Licence soulignent cependant une meilleure capacité à initier un questionnement et une plus grande liberté à émettre des hypothèses. Cet enthousiasme initial se trouve très rapidement mis à mal par l'absence de méthode de résolution et une incapacité à mettre en œuvre le formalisme scientifique. 
La situation semble encore plus préoccupante en IUT. Les répondants constatent que le raisonnement se restreint à l'application aveugle d'une formule. Les démonstrations n'intéressent pas les étudiants. On signale également un manque crucial de travail personnel.

\section{Prise d'initiative et participation}

En CPGE et en Licence, beaucoup de répondants notent une prise de parole plus facile. Malheureusement, ce point qui devrait être positif, doit être tempéré par l'absence de bases théoriques pour pousser la démarche au-delà du simple questionnement. La participation des étudiants porte souvent sur des questions générales ("culture »), plutôt que sur des points précis nécessitant une argumentation.

En IUT, selon la majorité des réponses, les étudiants sont de plus en plus passifs, plutôt consommateurs, et ne témoignent pas d'intérêt pour les nouvelles disciplines enseignées.

\section{Aptitudes expérimentales}

Une évolution des aptitudes expérimentales semble plus marquée en CPGE qu'en IUT et surtout qu'en Licence.

Globalement, on observe une amélioration de l'autonomie des étudiants devant la mise en œuvre d'un protocole expérimental. Ils ont moins d'inhibition à manipuler des appareils inconnus et ils prennent plus d'initiatives. En revanche, la rédaction d'un compte-rendu est très difficile. Certains étudiants donnent l'impression de n'en avoir jamais fait. Les notions d'incertitude leur sont souvent étrangères.

\section{Démarche et culture scientifiques}

En CPGE et en Licence, les réponses sont quasi unanimes : la culture générale des étudiants est plus étendue, mais plus superficielle. Ils ont souvent une image faussée de la physique, plus technologique que physique. Le vocabulaire utilisé est fréquemment erroné. Ils pensent savoir beaucoup sur beaucoup de choses, mais ils sont incapables d'expliquer, pour en avoir entendu parler de manière "trop vague ". Il semble que cet état de fait engendre chez les étudiants un manque de confiance, en particulier vis-à-vis de la résolution d'exercices ou de problèmes. En IUT, les étudiants ont quelques éléments de culture scientifique, difficiles à quantifier.

La formation à la démarche scientifique est clairement insuffisante et en régression. Le manque d'heures est cité comme l'une des explications. La physique ne semble désormais être perçue que comme un catalogue de problèmes et de formules pour les résoudre, sans aucun lien entre eux.

\section{Aptitudes à communiquer}

Un peu plus d'un tiers des répondants, quelle que soit la filière d'enseignement supérieur, indique avoir observé des évolutions dans les aptitudes des étudiants à communiquer.

Ces derniers semblent bien mieux maitriser la communication orale, tout au moins dans son aspect formel. Ils participent davantage, mais leur sens critique est moins développé et ils éprouvent plus de difficultés à aller à l'essentiel.

En revanche, l'expression écrite est de moins bonne qualité (certains répondants disent même " catastrophique »), tant dans la forme (orthographe, grammaire) que dans le fond (absence de capacité de synthèse, d'analyse critique des résultats...).

\section{Adéquation des programmes du lycée en physique et en mathématiques avec l'enseignement supérieur}

La quasi-totalité des répondants souligne l'inadéquation entre les programmes du secondaire en physique et en mathématiques et le niveau requis dans l'enseignement supérieur (fig. 3).

Le principal souhait formulé par les enseignants de CPGE est la restriction des domaines étudiés au profit d'un retour à l'approfondissement. Le "vernis " culturel se fait au détriment de l'acquisition de bases solides, et les outils mathématiques sont trop peu utilisés.

Pour les répondants d'IUT, il manque clairement une année de consolidation entre la terminale et la première année d'IUT.

En Licence, de nombreuses universités sont amenées à construire leur première année à la manière d'une terminale scientifique " ancienne formule".

Un point très important a été plusieurs fois souligné. Alors que les nouveaux programmes ont clairement affiché pour objectif de permettre aux futurs étudiants de mieux choisir leur orientation, on constate que beaucoup d'entre eux arrivent avec une idée complètement fausse de ce qu'est la physique. Ils appréhendent celle-ci comme une matière à la frontière du littéraire, pour laquelle les connaissances se résument à des descriptions de situations et à l'application de formules pour interpréter ces dernières. Le choc ressenti par nombre d'étudiants est important lorsqu'ils constatent que rigueur méthodologique et formalisme mathématique avancé sont les outils quotidiens du physicien.

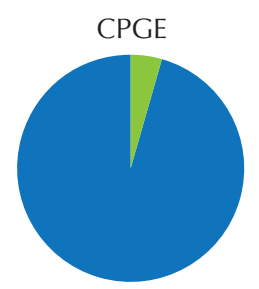

oui : $4,5 \%$ non : $95,5 \%$

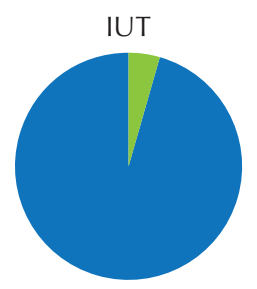

oui : $4,5 \%$ non : $95,5 \%$

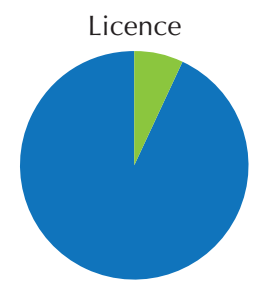

oui : $7,1 \%$ non : $92,9 \%$
3. Répartition des avis des répondants à la question : «Selon vous, est-ce que les programmes du lycée en mathématiques et en physique sont adéquats au niveau requis par l'Université, les IUT, les classes préparatoires? ”

\section{Pratique enseignante}

L'enquête montre que la plupart des répondants, toutes filières confondues, ont modifié leur enseignement suite à la réforme des programmes de lycée, avec un pourcentage plus élevé en CPGE $(96,6 \%)$.

Du point de vue des contenus, une majorité d'enseignants a été contrainte de renforcer très sérieusement les enseignements en mathématiques pour physiciens. C'est une conséquence probable de la cassure très nette apparue dans les programmes du lycée entre mathématiques, pour lesquelles un certain formalisme est maintenu, et sciences physiques, dont l'approche culturelle fait qu'elles sont de plus en plus identifiées comme "matière littéraire " par les étudiants.

Il apparaît que les enseignants ont été obligés d'adapter fortement leurs cours afin d'éveiller la curiosité des étudiants, désormais habitués à vivre la physique en spectateur/consommateur. 


\section{Conclusion}

Les programmes actuels mis en place depuis 2010 dans l'enseignement secondaire, ont été construits pour permettre aux élèves de mieux comprendre les enjeux sociétaux. C'est ainsi que l'aspect culturel de la science y prend une place de plus en plus importante. Cette position peut paraître intéressante au premier abord. Cependant, dans le cas de la physique, ce qui ressort de l'enquête de la SFP montre que les étudiants maîtrisent moins bien les concepts et lois, raisonnent de manière approximative et ne possèdent pas une culture scientifique aussi développée que ce que l'on aurait pu attendre.

Selon le ministère de l'Éducation nationale, les nouveaux programmes de physique au lycée n'ont plus vocation à former des scientifiques. Mais, selon la SFP, attendre la première année du supérieur pour former des scientifiques est trop tardif. Le risque de ces programmes est de ne plus former les scientifiques de qualité dont la France a besoin, notamment en recherche et développement, pour innover et permettre aux entreprises françaises d'être compétitives au niveau international. Citons un extrait de l'enquête de 2012 réalisée par l'Institute of Physics britannique : "[...] Les entreprises qui sont tributaires de la physique contribuent davantage à l'économie nationale que le secteur de la construction, emploient plus de personnes que le secteur de la finance... [Elles] contribuent pour $8,5 \%$ à la production économique du Royaume-Uni et emploient plus d'un million de personnes. "Une enquête de la Société Européenne de Physique (EPS) a conduit à des conclusions voisines.

Dans une lettre adressée à la ministre de l'Éducation nationale en date du 6 janvier 2014, la SFP a exprimé son inquiétude sur l'impact de ces nouveaux programmes de physique sur l'enseignement supérieur. Les résultats de l'enquête présentée ici ne font que renforcer cette inquiétude, et montrent l'urgence de retravailler sur les programmes pré-bac, de manière à ce qu'ils soient adaptés à l'entrée dans l'enseignement supérieur scientifique.

Face à ces constats, un groupe de travail interassociations (SFP, UdPPC, UPS) a été créé en 2014 pour mener une réflexion sur l'enseignement de la physique, afin de former les futurs citoyens et scientifiques dont la société a besoin, c'est-à-dire des hommes et des femmes ayant un esprit critique, sachant raisonner et possédant une bonne culture scientifique. L'objectif de ce groupe d'experts est de proposer au Conseil supérieur des programmes (CSP) une révision des programmes de première et terminale $S$, et au préalable de ceux de seconde, à l'horizon de la rentrée 2016 .

(a) UdPPC : Union des Professeurs de Physique et de Chimie

(b) UPS : Union des Professeurs de classes préparatoires Scientifiques

Nathalie Lebrun ${ }^{(1)}$ (nathalie.lebrun@univ-lillel.fr), Rémi Barbet-Massin ${ }^{(2)}$, Sylvie Magnier ${ }^{(3)}$ et Denis Dumora ${ }^{(4)}$

(1) Maître de conférences, Université de Lille et LDAR, Université Paris Diderot

(2) Professeur de physique en CPGE, Lycée Henri IV, Paris

(3) Professeur des universités, IUT A et Laboratoire PhLAM, Université de Lille

(4) Professeur des universités, CEN de Bordeaux Gradignan et Université de Bordeaux 